\title{
Comparison Between the NIST and the KEBS for the Determination of Air Kerma Calibration Coefficients for Narrow X-Ray Spectra and ${ }^{137}$ Cs Gamma-Ray Beams
}

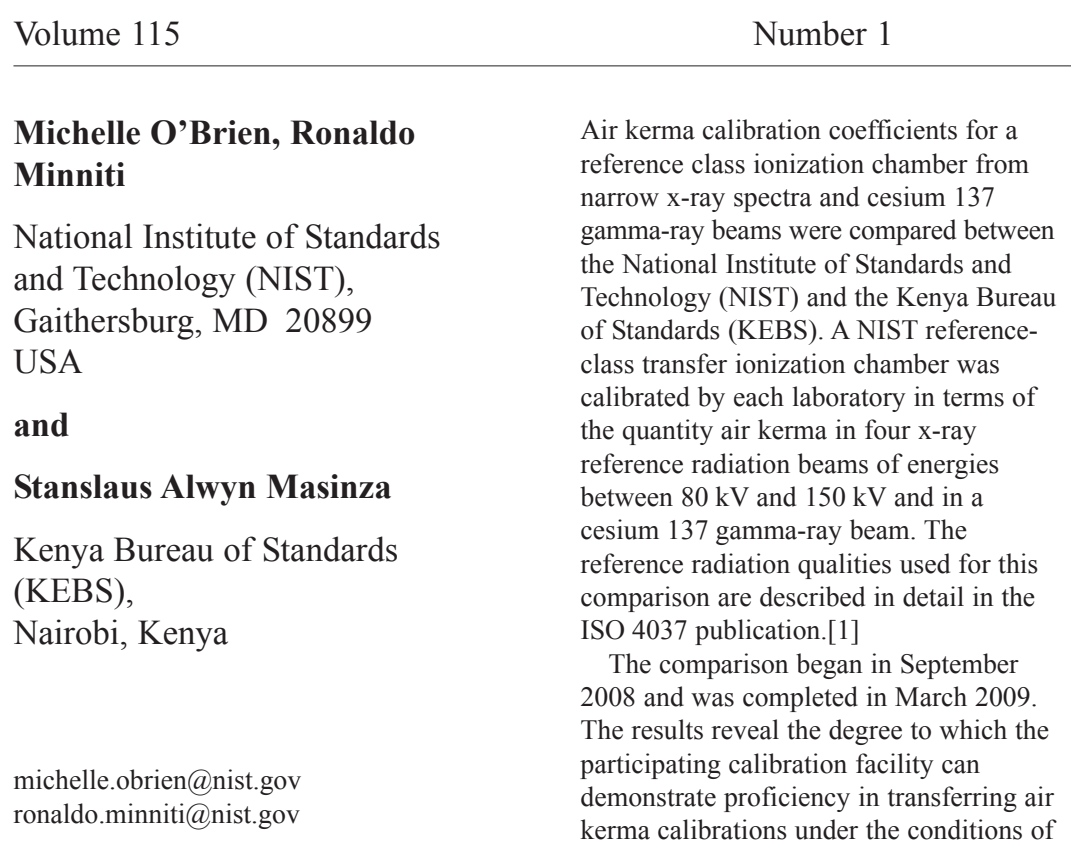

the said facility at the time of the measurements. The comparison of the calibration coefficients is based on the average ratios of calibration coefficients.

Key words: air kerma; free-air ionization chamber; gamma-ray, cesium 137; primary standard; reference radiation qualities; $\mathrm{x}$-ray calibration.

Accepted: January 20, 2010

Available online: $h$ ttp://www.nist.gov/jres

\section{Introduction}

The objective of this proposal was to compare the air kerma calibration coefficients of a reference class chamber determined in reference beams of $\mathrm{x}$ rays and gamma rays. The comparison was performed between the National Institute of Standards (NIST) and the Kenya Bureau of Standards (KEBS). A NIST referenceclass transfer ionization chamber was calibrated by each laboratory in terms of the quantity air kerma in four ISO narrow x-ray spectra beams with energies between $80 \mathrm{kV}$ and $150 \mathrm{kV}$ and in a ${ }^{137} \mathrm{Cs}$ gamma-ray beam. The reference radiation qualities used for this comparison are described in detail in the ISO 4037 publication [1].

\section{Procedure}

\subsection{Object of Comparison}

The object was to compare the value of the air kerma calibration coefficient of the chamber determined at the KEBS with the value obtained at the NIST. The transfer chamber used during the comparison was an Exradin $^{1}$ chamber model A5, SN XY051605.

\footnotetext{
${ }^{1}$ Certain commercial equipment, instruments, or materials are identified in this paper to foster understanding. Such identification does not imply recommendation or endorsement, nor does it imply that the equipment identified are necessarily the best available for the purpose.
} 


\subsection{Transfer Chamber}

The chamber used is spherical and made of C552 air equivalent plastic and has a nominal volume of $100 \mathrm{~cm}^{3}$. The air kerma calibration coefficient $N_{K}$ was determined by evaluating the ratio $N_{K}=K_{\text {air }} / I_{\text {corr }}$, where $K_{\text {air }}$ is the reference air kerma rate (expressed in units of $\mathrm{Gy} / \mathrm{s}$ ) and $I_{\text {corr }}$ is the measured ionization current (expressed in units of $\mathrm{C} / \mathrm{s}$ ) corrected for influence quantities. A bias voltage of 400 volts was applied to the chamber. This is the maximum voltage allowed by the electrometer owned by the KEBS. Table 1 summarizes the chamber specifications.

Table 1. Description of the chamber

\begin{tabular}{cccccc}
\hline \hline Type & $\begin{array}{c}\text { Serial } \\
\text { Number }\end{array}$ & $\begin{array}{c}\text { Sensitive } \\
\text { volume } \\
\text { (nominal }\end{array}$ & $\begin{array}{c}\text { Outside } \\
\text { Diameter } \\
\text { (nominal) }\end{array}$ & $\begin{array}{c}\text { Diameter } \\
\text { of inner }\end{array}$ & electrode \\
\hline Exradin A5 & XY051605 & $100 \mathrm{~cm}^{3}$ & $6 \mathrm{~cm}$ & $6 \mathrm{~mm}$ & $400 \mathrm{~V}$ \\
\hline
\end{tabular}

\subsection{Reference Radiation Qualities}

The measurements made at the NIST were performed in the x-ray and gamma-ray beam calibration facilities. The air kerma rates at both of these facilities are well established and are determined using the NIST primary standard instruments. Details on the determination of the air kerma rates using the primary standard instruments and the NIST calibration service can be found in Refs. [2-5].

For the x-ray measurements, the air kerma rates were determined directly using the Wyckoff-Attix free-air chamber. This primary standard for $\mathrm{x}$ rays is located in the NIST $300 \mathrm{kV}$ calibration facility. The $\mathrm{x}$-ray source used at the time of the comparison was a $320 \mathrm{kV}$ x-ray generator with a metal ceramic $\mathrm{x}$-ray tube, both supplied by Seifert-Pantak. ${ }^{1}$ The $\mathrm{x}$-ray generator is a high-frequency, highly stabilized voltage source. The tungsten anode X-ray tube, model MXR-321, has a beryllium window of thickness $3 \mathrm{~mm}$ and a focal spot $8.0 \mathrm{~mm}$ in diameter. The materials used for the filtration and for the measurement of Half Value Layer (HVL) were at least $99.99 \%$ pure with thicknesses known with an uncertainty of $0.01 \mathrm{~mm}$. The reference radiation qualities used for the comparison are

\footnotetext{
${ }^{1}$ Certain commercial equipment, instruments, or materials are identified in this paper to foster understanding. Such identification does not imply recommendation or endorsement, nor does it imply that the equipment identified are necessarily the best available for the purpose.
}

described in ISO 4037 as the narrow spectra [1]. The NIST and the KEBS HVL's are listed in Table 2.

Table 2. Characteristics of reference radiation qualities

\begin{tabular}{|c|c|c|c|}
\hline \multirow{3}{*}{$\begin{array}{l}\text { Reference } \\
\text { Radiation }\end{array}$} & \multirow{3}{*}{$\begin{array}{l}\text { Generating tube potential } \\
\qquad(\mathrm{kV})\end{array}$} & NIST & KEBS \\
\hline & & & \\
\hline & & \multicolumn{2}{|c|}{ Half-value layer ( $\mathrm{mm} \mathrm{Cu}$} \\
\hline NS80 & 80 & 0.59 & 0.56 \\
\hline NS100 & 100 & 1.14 & 1.07 \\
\hline NS120 & 120 & 1.76 & 1.67 \\
\hline NS150 & 150 & 2.41 & 2.34 \\
\hline Cs-137 & Not Applicable & 10.8 & 10.9 \\
\hline
\end{tabular}

The gamma-ray measurements were made in the NIST ${ }^{137} \mathrm{Cs}$ horizontal beam gamma-ray calibration facility. The air kerma rates in this facility were obtained by applying a decay correction to the reference air kerma rate. The reference air kerma rates were determined using a suite of six graphite wall cavity ionization chambers. The beam is collimated providing a circular beam size of $15 \mathrm{~cm}$ radius at a source-todetector distance of $195 \mathrm{~cm}$. The beam is uniform over the area across the chamber used in this work. Various types of reference class ionization chambers are used routinely to track the reference air kerma rate value.

\subsubsection{Reference Conditions at the NIST}

At the NIST each x-ray calibration was made by alternating between the transfer chambers and the standard free-air chamber, through the translation of the chambers to one beam center line. Beam alignment to the axis is accepted to be $0.1 \mathrm{~mm}$ and reproducible to better than $0.01 \mathrm{~mm}$, as observed by an alignment telescope. The reference plane for all measurements at the NIST was positioned at $1000 \mathrm{~mm}$ from the $\mathrm{x}$-ray radiation source and $1950 \mathrm{~mm}$ from the ${ }^{137} \mathrm{Cs}$ source. The beam diameter in the reference plane was at least $90 \mathrm{~mm}$ for the $\mathrm{x}$-ray measurements and at least $150 \mathrm{~mm}$ for the gamma-ray measurements. The exact beam geometry was not prescribed in the protocol so that the KEBS could use the appropriate geometry depending on the field size and field uniformities available and the conditions that best represents routine calibrations at their facility. The influence of field size was not investigated for this comparison. The geometry and conditions of the air kerma measurement were kept consistent at NIST for all measurements. Table 3 describes the radiation reference field sizes used for the comparison. 
Table 3. Beam geometry used at both facilities

\begin{tabular}{lcccr}
\hline \multicolumn{1}{c}{ Geometry } & NIST & KEBS & NIST & KEBS \\
& X-ray spectra & ${ }^{137} \mathrm{Cs}$ & \\
\hline Calibration distance (mm) & 1000 & 2000 & 1950 & 1950 \\
Beam diameter (mm) & 90 & 853 & 150 & 682.5 \\
\hline
\end{tabular}

At the NIST the chamber background current was measured before and after the chamber was exposed to the source of radiation. The average of the background currents was determined and subtracted from the measured ionization current produced in the chamber when exposed to the source of radiation. Typical background currents were of the order of $0.01 \%$ of the ionization current. The integration times used at the NIST were 120 seconds for the $\mathrm{x}$-ray measurements and 60 seconds for the gamma-ray measurements. The measured ionization currents were normalized to $295.15 \mathrm{~K}$ and $101325 \mathrm{~Pa}$. The humidity was monitored and recorded and was typically in a range of $30 \%$, however the currents were not corrected for humidity. Only the negative polarity measurements were used for the comparison results, resulting in a negative charge.collected on the electrometers. No correction was applied to the calibration coefficient to account for polarity effect since at both facilities the same polarity was used. The NIST performed measurements at both polarities to verify the influence of the polarity effect. The value of the calibration coefficients obtained with both polarities agreed within less than $0.10 \%$ for all reference beams used. This implies that the polarity effect is negligible. No corrections were applied to the calibration coefficients to account for electronic recombination effects. However an estimate of the recombination correction was obtained using the two-voltage method. For this, the ratio of the ionization currents measured at the full voltage of $400 \mathrm{~V}$ and half voltage of $200 \mathrm{~V}$ was obtained.

The KEBS was requested to provide the calibration coefficients for the transfer chambers in terms of air kerma per charge in units of $\mathrm{Gy} / \mathrm{C}$ and refer to standard conditions of air temperature at $295.15 \mathrm{~K}$, pressure at $1013.25 \mathrm{hPa}$ and $50 \%$ relative humidity. The KEBS was requested to use the same polarity for the voltage bias of the Exradin chamber.

\subsubsection{Reference Conditions at the KEBS}

X-ray calibrations for each beam quality were made by alternating between the transfer chambers and the standard free-air chamber, through the translation of the chambers to the central beam axis indicated by the laser beam. The details of the KEBS standard can be found in Ref. [6]. The x-ray apparatus at KEBS consists of several elements placed between the $\mathrm{X}$-ray tube and the detection distance. Starting at the $\mathrm{x}$-ray source these elements include a diaphragm/aperture wheel holder at $200 \mathrm{~mm}$ from the source, a filter wheel holder at $220 \mathrm{~mm}$, a permanent tube inherent filter holder at $230 \mathrm{~mm}$, a monitor chamber at $240 \mathrm{~mm}$, and a HVL filter holder. The HVL diaphragm holder is only used for HVL measurements and is placed at $630 \mathrm{~mm}$ from the tube. The diaphragm holder is remotely adjustable from the control unit. The filters can be selected from the control unit. The comparison ionization chamber was placed at the measuring distance of $2000 \mathrm{~mm}$ from the source. Details of the KEBS laboratory setup can be found in Ref. [7].

The gamma-ray measurements were made in the KEBS ${ }^{137} \mathrm{Cs}$ gamma-ray calibration laboratory. The air kerma rates in this facility are obtained by applying a decay correction to the reference air kerma rates that were established on July 2007, at the time the facility was commissioned. These daily air kerma rate values are tracked using a laboratory developed spreadsheet and confirmed through routine measurements using ionization chambers models LS01 and LS10 as part of the quality control of the facility. It is important to mention that the air kerma standards at KEBS are traceable indirectly to the Bureau International des Poids et Measures (BIPM) standards through the International Atomic Energy Agency (IAEA). The reference air kerma rates at KEBS were determined using the KEBS secondary standard chamber model W-32002, SN 247 calibrated at the International Atomic Energy Agency (IAEA). The value of the most recent ${ }^{137} \mathrm{Cs}$ calibration coefficient of the KEBS standard chamber obtained from IAEA is $2.52 \times 10^{4} \mathrm{~Gy} / \mathrm{C}$. This value includes a correction introduced by the BIPM in the year 2009 that applies to all calibration facilities that are directly and indirectly traceable to the BIPM, including KEBS. Furthermore, all results presented in this work and obtained at KEBS, include all corrections introduced by the BIPM up to the date of this publication.

The KEBS gamma beam geometry uses a collimated circular beam with a radius of approximately $340 \mathrm{~mm}$ at a source-to-detector distance of $1950 \mathrm{~mm}$. The beam is uniform over the area across the chamber. At a distance of $1000 \mathrm{~mm}$, the variation of the beam intensity across a circular area of $2850 \mathrm{~mm}$ does not exceed $5 \%$ when the largest collimator is used. 
The NIST chamber was connected to the PTW electrometer. Prior to performing the measurements, a voltage was applied to the chamber overnight to allow sufficient time for the chamber to stabilize. Background currents were measured before and after the chamber was exposed to the source of radiation. The average of the background currents was determined and subtracted from the measured ionization current produced in the chamber when exposed to the source of radiation. Typical background currents were of the order of $0.02 \%$ of the ionization current. The integration time used at the KEBS was 60 seconds for both the x-ray and the gamma-ray measurements. The measured ionization currents were normalized to 295.15 K and $101325 \mathrm{~Pa}$. The humidity was monitored and recorded and was typically in a range of $40 \%$, however the currents were not corrected for humidity. The calibration coefficients for the transfer chamber were given in terms of air kerma per charge in units of $\mathrm{Gy} / \mathrm{C}$ and corrected to standard conditions.

\subsection{Course of Comparison}

The NIST shipped the chamber to the KEBS in September 2008 with a tentative scheduled return to the NIST of November 2008. However, due to unexpected delays at the KEBS resulting from equipment failure the measurements were completed in March 2009. After completion of the calibrations at the KEBS facility and the return of the chamber to the NIST, constancy checks were performed in late March and early April of 2009. The values of the calibration coefficients obtained at the NIST before and after the shipment of the chamber to the KEBS agreed as expected, indicating that there were no alterations or damage to the chamber during the full period of the comparison.

\section{Uncertainties}

The uncertainties associated with the NIST primary $\mathrm{x}$-ray standard are listed in Table 4. Following standard guidelines for the expression of uncertainity published elsewhere [8,9], the uncertainties listed in the tables in this work are classified in Type A and Type B according to the method used to evaluate them. Type A uncertainties, denoted as $u_{i \mathrm{~A}}$, are determined from statistical analysis while Type B uncertainties, denoted as $u_{i \mathrm{~B}}$, are determined by other means such as scientific judgment. As shown in the table, the relative fractional combined uncertainty of the air kerma rate, $\dot{K}_{\text {Standard }}$, from the $\mathrm{X}$-ray beam is 0.0030 . Similarly, the relative combined
Table 4. Uncertainties associated with the NIST standard for the $\mathrm{x}$-ray spectra

\begin{tabular}{lcc}
\hline \hline & \multicolumn{2}{c}{ NIST standard } \\
Relative standard uncertainty & $u_{i \mathrm{~A}}$ & $u_{i \mathrm{~B}}$ \\
\hline Ionization current & 0.0008 & 0.001 \\
Volume & 0.0004 & 0.0001 \\
Positioning & & 0.0001 \\
Correction factors (excl. $\left.k_{\mathrm{h}}\right)$ & 0.0012 & 0.0024 \\
Humidity $k_{\mathrm{h}}$ & & 0.0003 \\
Physical constants & & 0.0015 \\
\hline$\dot{K}_{\text {Standard }}$ & 0.0015 & 0.003 \\
\hline
\end{tabular}

uncertainty for the air kerma rate, $\dot{K}_{\text {Standard }}$, from the ${ }^{137} \mathrm{Cs}$ gamma ray beam is 0.0029 (as listed in Table 7 ahead). This results in relative combined uncertainties of the air kerma rates of $0.30 \%$ and $0.29 \%$ for $\mathrm{x}$ rays and gamma rays respectively when expressed as a percentage. Table 5 and Table 6 show the uncertainty analysis associated with the calibration of the transfer chamber at the NIST and the KEBS using $x$ rays. The uncertainty of the calibration is given by the uncertainty of the calibration coefficient $N_{K}$. Similarly Table 7 and 8 show the uncertainty analysis associated with the calibration of the chamber at both facilities in the ${ }^{137} \mathrm{Cs}$ beam. Note that in Tables 7 and 8, the relative uncertainties of the chamber current corrected for influence quantities and the air kerma rate value provided by the standard, are combined, resulting in the relative standard uncertainty of the calibration coefficient $N_{K}$. Finally, Table 9 lists the relative combined uncertainty of the comparison ratio, KEBS/ NIST, obtained both with $\mathrm{x}$-rays and gamma-rays. The uncertainty of the calibration coefficients $N_{K}$ for the x-ray portion of the comparison listed in Table 9 are grouped in Type A and Type B evaluations, whereas the uncertainties for the gamma ray calibration coefficients are expressed directly as the relative combined uncertainties.

Table 5. Uncertainties associated with the NIST calibration of the A5 Exradin transfer chamber in the x-ray beam

\begin{tabular}{llr}
\hline \hline & \multicolumn{2}{c}{ NIST standard } \\
Relative standard uncertainty & $u_{i \mathrm{~A}}$ & $u_{i \mathrm{~B}}$ \\
\hline$\dot{K}_{\text {Standard }}$ & 0.0015 & 0.0030 \\
Ionization current & 0.00083 & 0.0010 \\
Positioning & 0.0001 & 0.0003 \\
Humidity & & 0.0032 \\
$N_{K, \text { NIST }}$ & 0.0015 & 0.00354 \\
\hline
\end{tabular}


Volume 115, Number 1, January-February 2010

Journal of Research of the National Institute of Standards and Technology

Table 6. Uncertainties associated with the KEBS calibration of the A5 Exradin transfer chamber in the x-ray beam

\begin{tabular}{lcc}
\hline \hline & & KEBS \\
Relative standard uncertainty & $u_{i \mathrm{~A}}$ & $u_{i \mathrm{~B}}$ \\
& KEBS Reference chamber & \\
$N_{K, \text { reference }}$ & 0.00200 & \\
Long term stability & 0.00052 & 0.00290 \\
Positioning & & \\
Ionization current & 0.00210 & \\
Temperature and pressure & 0.00003 & 0.00290 \\
& Exradin chamber at KEBS & \\
Positioning & & \\
Temperature and pressure & 0.00070 & 0.00410 \\
Ionization current & 0.00667 & \\
\hline$N_{K, \text { KEBS }}$ & 0.00732 & 0.00839 \\
\end{tabular}

Table 7. Uncertainties associated with the NIST calibration of the A5 Exradin transfer chamber in the ${ }^{137} \mathrm{Cs}$ gamma-ray beam

\begin{tabular}{|c|c|c|}
\hline \multirow[b]{2}{*}{ Relative standard uncertainty } & \multicolumn{2}{|c|}{ NIST } \\
\hline & $u_{i \mathrm{~A}}$ & $u_{i \mathrm{~B}}$ \\
\hline Charge & 0.0010 & 0.0010 \\
\hline Time & & 0.0005 \\
\hline Air density correction (temperature and pressure) & & 0.0003 \\
\hline Distance & & 0.0002 \\
\hline$k_{\text {sat }}$, loss of ionization due to recombination & 0.0001 & 0.0005 \\
\hline Probe orientation & & 0.0001 \\
\hline Humidity & & 0.0006 \\
\hline & 0.0010 & 0.0014 \\
\hline \multicolumn{3}{|l|}{ Chamber current corrected for all influence quantities } \\
\hline & \multicolumn{2}{|c|}{0.0017} \\
\hline$\dot{K}_{\text {Standard }}$ & \multicolumn{2}{|c|}{0.0029} \\
\hline$N_{K, \mathrm{NIST}}$ & \multicolumn{2}{|c|}{0.0034} \\
\hline
\end{tabular}

Table 8. Uncertainties associated with the KEBS calibration of the A5 Exradin transfer chamber in the ${ }^{137}$ Cs gamma-ray beam

\begin{tabular}{|c|c|c|}
\hline & \multicolumn{2}{|c|}{ KEBS } \\
\hline Relative standard uncertainty & $u_{i \mathrm{~A}}$ & $u_{i \mathrm{~B}}$ \\
\hline Stability of reference value $K_{\text {air }}$ at $2 \mathrm{~m}$ & 0.0024 & 0.0000 \\
\hline Temperature and pressure & 0.0003 & 0.0015 \\
\hline Leakage current & 0.0000 & 0.0100 \\
\hline & 0.0024 & 0.0 .0101 \\
\hline \multicolumn{3}{|c|}{ Chamber current corrected for all influence quantities } \\
\hline & \multicolumn{2}{|c|}{0.0104} \\
\hline$\dot{K}_{\text {Standard }}$ & \multicolumn{2}{|c|}{0.0078} \\
\hline$N_{K, \mathrm{NIST}}$ & \multicolumn{2}{|c|}{0.0130} \\
\hline
\end{tabular}


Table 9. Uncertainties associated with the comparison results

\begin{tabular}{lccc}
\hline \hline & & X-rays & Cs- 137 \\
Relative standard uncertainty & $u_{i \mathrm{~A}}$ & $u_{i \mathrm{~B}}$ & $u_{i}$ \\
\hline$N_{K, \mathrm{KEBS}}$ & 0.0073 & 0.0041 & 0.0130 \\
$N_{K, \mathrm{NIST}} / N_{K, \text { NIST }}$ & 0.0015 & 0.0032 & 0.0034 \\
$N_{K, \mathrm{KEBS}}$ & 0.0075 & 0.0052 & 0.0134 \\
\hline$\dot{K}_{\mathrm{KEBS}} / \dot{K}_{\mathrm{NIST}}$ & & 0.0091 & \\
\hline
\end{tabular}

\section{Results and Discussion}

The mean value of the calibration coefficients $N_{K}$ measured at the NIST and the KEBS are shown in Table 10. The average air kerma rates used at both facilities are listed in Table 11. The comparison results are listed in Table 12 as the ratio of the mean calibration coefficients, $N_{K, \mathrm{KEBS}} / N_{K, \mathrm{NIST}}$ for each reference radiation with an associated comparison uncertainty listed in Table 9. The mean ratio of the comparison ratios for the $\mathrm{x}$ rays is 0.984 . The corresponding standard deviation of the four comparison ratios is 0.0083. Acceptable agreement is also achieved for the gamma-ray comparison with a resulting ratio of 1.006 with a standard uncertainty of the ratio of 0.0134 .

The differences observed in the values of the calibration coefficients, resulting in agreement of approximately $1.6 \%$ for $\mathrm{x}$ rays and $0.6 \%$ for gamma rays, can be due to the variations in the field sizes and source to detector distances used at both facilities. Although the beam uniformity and scatter characteristics at NIST meet the conditions as described in the ISO 4037 [1] , measurements at the NIST, using the Exradin A5 chamber at various distances and field sizes, result in differences of the calibration coefficient of up to $1 \%$. Since this comparison was not designed to compare beam geometry, the uncertainty due to differences in field sizes and scatter conditions was not directly addressed.

The polarity effect and the electronic recombination effect were excluded as possible influences to the comparison results. The differences due to the polarity of the comparison chamber bias voltage were measured to have a negligible effect for the specific chamber volume and the rates used for the comparison. The air kerma rates used at both laboratories were similar, resulting in probable similar electronic recombination effects. The estimate of the recombination effect obtained using the two-voltage method resulted in negligible influences. The uncertainty analysis did not include these negligible components.
Table 10. Mean values of the calibration coefficients measured at the NIST and the KEBS using the Exradin A5

\begin{tabular}{ccc}
\hline & \multicolumn{2}{c}{ Calibration coefficients } \\
& \multicolumn{2}{c}{$10^{5} \mathrm{GyC}^{-1}$} \\
Reference radiation & NIST & KEBS \\
& Mean $N_{K}$ & Mean $N_{K}$ \\
\hline NS100 & 2.969 & 2.886 \\
NS80 & 2.929 & 2.898 \\
NS100 & 2.969 & 2.886 \\
NS120 & 2.999 & 2.961 \\
NS150 & 2.991 & 2.955 \\
Cs-137 & 3.047 & 3.065 \\
\hline
\end{tabular}

Table 11. Average air kerma rates used at both facilities

\begin{tabular}{ccc}
\hline \hline & \multicolumn{2}{c}{ Average air kerma rate } \\
$\begin{array}{c}\text { Reference radiation } \\
\text { Beam }\end{array}$ & NIST & KEBS \\
\hline NS80 & 6.61 & 3.74 \\
NS100 & 4.08 & 2.87 \\
NS120 & 4.50 & 1.59 \\
NS150 & 3.53 & 12.9 \\
Cs-137 & 26.0 & 3.31 \\
\hline
\end{tabular}

Table 12. Results of comparison

\begin{tabular}{cc}
\hline \hline Reference radiation & $\begin{array}{c}\text { Mean } \\
N_{K, \mathrm{KEBS}} / N_{K, \mathrm{NIST}}\end{array}$ \\
\hline $\mathrm{NS} 80$ & 0.990 \\
$\mathrm{NS} 100$ & 0.972 \\
$\mathrm{NS} 120$ & 0.987 \\
$\mathrm{NS} 150$ & 0.988 \\
Cs-137 & 1.006 \\
\hline
\end{tabular}




\section{References}

[1] ISO/IS 4037-1: $1996 \mathrm{X}$ and gamma reference radiations for calibrating dosimeters and dose rate meters and for determining their responses as a function of photon energy-Part 1.: Radiation characteristics and production methods.

[2] P. Lamperti and M. O'Brien, Calibration of X-Ray and Gamma-Ray Measuring Instruments, NIST Special Publication 250-58 (2001).

[3] C. M. O'Brien, NIST Quality Manual, Ionizing Radiation Division QM-II, Procedure 3. Calibration of x-ray radiation detectors. Gaithersburg, MD, NIST (2004).

[4] R. Minniti, NIST Quality Manual, Ionizing Radiation Division QM-II, Procedure 4. Calibration of gamma-ray radiation detectors. Gaithersburg, MD, NIST (2004).

[5] R. Minniti, H. Chen-Mayer, S. M. Seltzer, S. M. Huq, L. Bryson, T. Slowery, J. A. Micka, L. A. DeWerd, N. Wells, W. F. Hanson, and G. S. Ibbott, Med Phys 33,1074 (2006).

[6] IAEA, Austria, Calibration of Radiation Protection Monitoring Instruments, STI/PUB/1074 (January 2000).

[7] A. S. Masinza, KEBS Laboratory Procedure, MET-LP-19/17, X-Calibration Procedures Quality Control in X-Ray, (2008).

[8] B. N. Taylor and C. E. Kuyatt, Guidelines for evaluating and expressing the uncertainty of NIST measurement results, NIST Technical Note 1297, Washington, DC: U.S. Government Printing Office (1994).

[9] International Organization for Standardization (ISO) Guide to the Expression of Uncertainty in Measurement, Geneva, Switzerland: ISO (1993).

About the authors: Michelle O'Brien and Ronaldo Minniti are physicists in the Ionizing Radiation Division, Radiation Interactions and Dosimetry Group of the NIST Physics Laboratory. Mrs. O'Brien, an expert in $x$-ray dose traceability, maintains the NIST primary $x$-ray standards, while conducting comparisons between other national measurement institutions and facilitating $x$-ray dose measurement quality standards domestically. Dr Minniti, an expert in gammaray dose traceability, maintains the NIST gamma-ray measurement capabilities, while conducting comparisons between other national measurement institutions and facilitating dose measurement quality standards domestically. Alwyn Stanslaus Masinza is a member of the Radiation Dosimetry Laboratory at KEBS where the national standards in the field of Ionizing Radiation are maintained. It is a Secondary Standard Dosimetry Laboratory (SSDL) and was established in 2007 as a result of the collaboration of International Atomic Energy Agency (IAEA) and the Government of the Republic of Kenya through the project KEN/6/016. 